\title{
Pharmacoepidemiology and the Elderly
}

\section{S. Andrade and J. Gurwitz}

\section{Introduction}

Pharmacoepidemiology employs the methods of epidemiology to study the frequency, determinants, and outcomes of drug therapy.

Types of pharmacoepidemiologic investigations involve the evaluation of:

- Adverse and beneficial drug effects;

- Medication utilization patterns;

- Drug effects on quality-of-life;

- Economic impacts of medication use.

Pharmacoepidemiology is becoming increasingly important with the aging of Western populations, due to the increased prevalence of medication use among older persons. In such patients, the evaluation of the adverse consequences of using multiple medications concomitantly is particularly important.

\section{Types of Pharmacoepidemiologic Studies}

Types of investigations encompassed within the discipline of pharmacoepidemiology include the evaluation of adverse and beneficial effects of drug therapies, medication utilization patterns, 
drug effects on the quality of life, and the economic impact of medication use.

\section{Adverse and Beneficial Effects of Drug Therapy}

Pharmacoepidemiologic methods are most commonly employed to study adverse drug effects using postmarketing surveillance data. Some notable examples include the associations between nonsteroidal anti-inflammatory drugs and serious gastrointestinal complications such as haemorrhage and perforation, ${ }^{1-8}$ nonsteroidal anti-inflammatory drugs and nephrotoxicity, 9,10 benzodiazepines and falls and hip fracture, ${ }^{11-13}$ and metoclopramide and parkinsonism. ${ }^{14}$ In addition to quantifying the association between a drug and a specific adverse effect, the interaction between these effects and the demographic characteristics (gender, age and race) of the patient, as well as the influence of comorbidity and concomitant drug use can be evaluated. ${ }^{15}$

Pharmacoepidemiologic studies have also been used to evaluate the beneficial effects of drug therapy, such as the protective effects of aspirin use ${ }^{16,17}$ or estrogen replacement therapy ${ }^{18-20}$ against the occurrence of coronary heart disease, and the benefits of inhaled steroids for preventing hospitalization for asthma. ${ }^{21}$

\section{Drug Utilization Studies}

Drug utilization studies are used to evaluate the frequency and appropriateness of drug therapy. Patterns of medication use may be determined over time in specific patient groups.

The data may be used to detect over- or under-utilization of medications or to examine drug distribution or expenditure issues. ${ }^{22}$ In addition, appropriateness of therapy may be evaluated according to factors such as indication, dose, specific patient characteristics, medical conditions, or concomitant drug use. Three examples of drug utilization studies with special relevance to the 
geriatric population are: (1) antipyschotic drug use in the nursing home setting to treat agitation in patients with dementia, (2) the use of thrombolytic therapy in elderly persons who suffer acute myocardial infarction; and (3) the use of opioid analgesics in the management of cancer pain.

\section{Antipsychotic Drugs}

For decades, the use of antipsychotic drugs has remained extensive in nursing homes in the United States. Although recent regulatory changes may have had some effect, antipsychotic drugs were, until recently, given to about one fourth of all U.S. nursing home residents. ${ }^{23} \mathrm{~A}$ few studies suggest that antipyschotic drugs may be effective in the treatment of agitation in geriatric patients with dementia, ${ }^{24}$ but the literature on this topic is both limited and ambiguous. Clear evidence, however, links the use of these drugs with extrapyramidal symptoms, gait instability, falls, and hip fractures. ${ }^{25-27}$

\section{Thrombolysis in Acute Myocardial Infarction}

While the possibility of disabling or fatal intracranial haemorrhage is always a concern in patients treated with thrombolytics, these risks must be balanced against the benefits in the management of elderly acute myocardial infarction patients. The risk of death after acute myocardial infarction increases sharply with advancing patient age, and the few trials that enrolled meaningful numbers of elderly patients have suggested a beneficial effect of thrombolysis on short-term mortality associated with acute myocardial infarction in this population. ${ }^{28}$ Although there is a larger proportionate reduction in mortality with thrombolytic treatment for younger patients, older patients are at higher absolute risk of dying from acute myocardial infarction. As a result, the absolute mortality reductions (net lives saved per 
1,000 patients treated) are similar for younger and older patients. ${ }^{29}$ The view now is that age itself should not be a factor when deciding whether or not to use thrombolytic therapy in the setting of acute myocardial infarction. ${ }^{30}$ Yet, the use of these agents in acute myocardial infarction declines considerably with advancing patient age. ${ }^{31}$

\section{Opioids for Pain Management in Cancer}

Despite guidelines for pain management in metastatic cancer, ${ }^{32}$ treatment is often inadequate. In a study of 1,308 outpatients with metastatic cancer being followed by oncologists, 769 patients reported experiencing pain, which $62 \%$ characterized as substantial. ${ }^{33}$ Patients with poor pain control reported that the pain interfered with function and their quality of life. Older age was an independent risk factor for inadequate pain management. Concerns have been raised that the elderly are at risk of undertreatment because of the underestimation of their sensitivity to pain, a misconception that they tolerate pain well, and a reluctance to prescribe adequate doses of opioid analgesics to older cancer patients.

\section{Pharmacoeconomic Studies}

Pharmacoepidemiologic methods are increasingly being used to evaluate the total costs associated with drug therapy. These studies include evaluations of the overall effects of drugs on the utilization of medical services and costs of total medical care. The rates and costs of beneficial and adverse drug effects may be quantified, including resource costs such as medication acquisition and administration, inpatient and outpatient visits, laboratory tests and procedures, transportation to medical care facilities, and pain and suffering of the patient. Such methods may be utilized to compare the cost effectiveness of two or more alternative 
therapies which have different ingredient costs and differ in efficacy. ${ }^{34}$ Studies evaluating the additional resource utilization associated with adverse drug events in hospitalized patients have assessed the costs of longer periods of hospital stay. ${ }^{35,36}$ This subject is discussed in greater detail in Chapter 3.

\section{Quality-of-life Studies}

Quality-of-life studies may be conducted to evaluate the influence of medication use on the patients' well-being, including such domains as social, emotional, and physical function. Studies have evaluated the quality-of-life impact of medications including treatments for hypertension, ${ }^{37,38}$ rheumatoid arthritis, ${ }^{39,40}$ HIV infection, ${ }^{41}$ and cancer. ${ }^{42-44} \mathrm{~A}$ variety of measurement instruments have been developed to assess health-related quality of life. These tools may measure multiple aspects of patient functioning for a variety of conditions, or may be specific to a particular disease state, patient population, or function. ${ }^{45}$ Experience with using these methods to assess the impact of drug therapy on qualityof-life in the elderly have been extremely limited and valid instruments to measure quality-of-life dimensions specifically in the elderly are few.

\section{Importance of Postmarketing Drug Surveillance}

Past tragedies with marketed drugs have emphasized the need to evaluate drug safety. Possibly the most well-known medication disaster worldwide occurred in the 1960s shortly after the marketing of thalidomide, a mild hypnotic drug. A substantial increase in phocomelia, a birth defect resulting in the absence of limbs or parts of limbs, was observed, which epidemiologic studies found to be caused by in utero exposure to thalidomide. ${ }^{46-48}$ This and other tragedies ${ }^{49,50}$ have resulted in the establishment of national and international committees and bureaus, as well as 
laws and amendments to monitor drug safety, requiring preclinical testing of drug safety. However, even with the stricter regulation around the preclinical evaluation of drug safety, a number of medications have been found to cause serious adverse effects that have only been detected after marketing. ${ }^{51-54}$

Because of the limitations of premarketing clinical trials, pharmacoepidemiologic studies have become increasingly important in postmarketing drug surveillance (after the time of drug marketing). At the time a drug is approved for marketing, generally only a few hundred to a few thousand individuals have been administered the agent in premarketing clinical trials, which are primarily designed to assess drug efficacy. This is not a sufficient number to detect rare adverse events. In addition, certain patient groups may be excluded from clinical trials, based on patient gender or age (see Figure 1.1). ${ }^{55}$ Thus, the experience

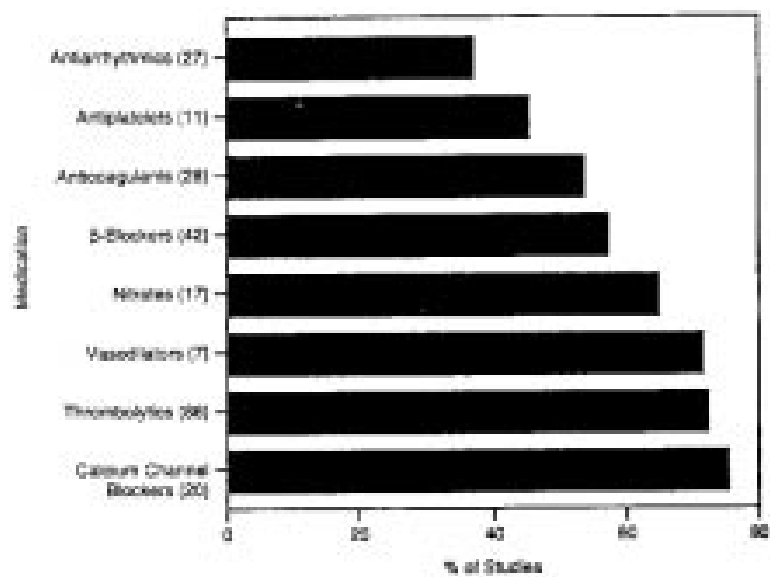

Figure 1.1. Percentage of clinical trials of pharmacotherapies employed in the treatment of acute myocardial infarction that excluded participation on the basis of patient age. All studies were published between 1960 and 1991. The numbers in parentheses indicate total number of studies.

(Source: Gurwitz et al., JAMA 1992) 
of drug use in population groups such as the elderly, who are often more seriously ill than patients included in the premarketing studies, is often unknown at the time of drug marketing. The effects of long-term use of agents (particularly those to treat chronic disorders) are generally unknown. Lastly, evaluation of the patterns and consequences of "typical" use in the population can only be evaluated once the drug is marketed. The prescribing patterns and economic impacts of medications may be difficult to predict before marketing of the drug, and can only be precisely assessed after marketing.

\section{Principles of Pharmacoepidemiologic Research}

\section{The Frequency of Adverse Drug Events}

The frequency of the occurrence of an adverse drug event is the fundamental measurement used in the evaluation of the distribution and risk factors for such an event. Measures of frequency commonly used in pharmacoepidemiologic studies are incidence and prevalence.

Incidence reflects the number of new cases of an adverse event developing over a specified period of time. Incidence measures are useful to assess differences in the probability of an adverse event in different populations and to study etiologic factors relevant to the occurrence of the event. Cumulative incidence, or risk, is one measure of incidence, and reflects the number of new cases of an event developing over a specified time period divided by the total population at risk of developing the event. Another measure of incidence is the incidence rate, or incidence density, which is a useful measure if individuals are followed for varying lengths of time. The incidence rate reflects the number of new cases of an event divided by the total sum of the time period at risk for each individual in the population, measured as persontime units (e.g., person-years). The person-time for each individual 
is the length of time the individual was a study subject and was free of the event of interest.

Prevalence reflects the proportion of people at a given point in time who have experienced an adverse drug event. The numerator of the prevalence estimate includes all existing cases of an event, rather than just new cases. The prevalence of an adverse drug event is a function of the incidence rate and the duration of the adverse drug event. If the prevalence of an adverse event is low and the incidence rate is constant over time, the prevalence equals the incidence rate multiplied by the duration of the adverse event.

\section{Study Designs}

Both experimental and observational designs are used in the performance of pharmacoepidemiologic studies. In experimental studies, the investigator allocates subjects to particular treatment regimens. Observational study designs are more commonly used in postmarketing surveillance studies. In observational studies, investigators do not control the allocation of the drug exposure; they identify and characterize the exposures and events in the individuals comprising specific populations.

\section{Observational Studies}

\section{Case Reports/Case Series}

Case reports and case series are descriptions of the experience of single patients or groups of patients who experience a given adverse drug event. The governments of many countries operate systems for collection of spontaneous reports of adverse drug events. These national systems vary in their organization, reporting requirements, and management of reports. For most countries, the reporting of suspected adverse drug events is 


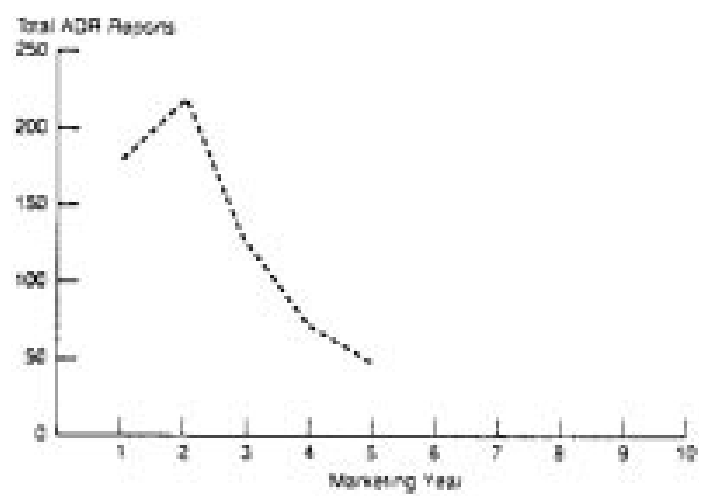

Figure 1.2. Spontaneous adverse drug reaction reports for seven nonsteroidal antiinflammatory drugs in the United Kingdom by year of marketing. Adverse drug reaction reporting is generally heaviest early in a product's life cycle. In this case, adverse drug reaction reporting is five times greater in the second versus the fifth marketing year.

(Source: Sachs et al., American Journal of Medicine 1986)

voluntary, ${ }^{56}$ with the majority received from health care professionals or pharmaceutical manufacturers.

While these systems are important for the generation of signals for problems with a medication not detected in pre-marketing studies, they are often limited by inadequate information to assess causality. More importantly, the amount of under-reporting is difficult to estimate, and may be selective, depending on the time since the drug has been on the market and publication of reports in the medical literature (see Figure 1.2). ${ }^{56}$

\section{Ecologic (correlational) Studies}

In ecologic studies, the occurrence of an event and of an exposure are compared over time or in different geographic regions. The unit of observation is a group of individuals, and the exposure and event status are not known at the individual level. A major 
limitation of ecologic studies is the lack of ability to control for potentially confounding factors. Thus, an observed relationship between a drug exposure and an adverse event may be due to another unmeasured or unappreciated factor.

\section{Cross-sectional Studies}

Cross-sectional studies determine exposure and event status at the same point in time. They provide information on the prevalence of an adverse drug event in specific populations. However, a major disadvantage of these studies is the difficulty in determining whether the drug exposure preceded or followed the occurrence of the event. This study design limitation compromises the ability to assess whether there is a causal association between a drug exposure and a potential adverse drug event.

\section{Case-control Studies}

In case-control studies, 'cases' who have suffered an adverse drug event of interest and 'controls' who have not suffered an event are compared with respect to their prior exposure status to a drug. Case-control studies are relatively quick and inexpensive to conduct compared to follow-up studies (prospective cohort studies or clinical trials), and they are useful for the studying of rare events or events with long latent periods. In addition, multiple risk factors for a given event may be evaluated. The main disadvantages of case-control studies relate to potential biases in the selection of appropriate control groups and differences in the recall of drug exposures between case and control patients. Studies based on retrospectively collected data may be limited by the completeness and validity of the information available. 


\section{Cohort Studies}

In cohort studies, individuals are classified with regard to their exposure status to a drug and are monitored over time to detect new cases of the adverse drug event. Cohort studies may be prospective or retrospective. In prospective study designs, the events of interest have not occurred at the time the study is initiated. In retrospective study designs, both the exposures and events of interest have occurred at the time of initiation of the study.

Because the individual's exposure status is determined prior to the classification of the event status, cohort studies are less prone to recall bias. Other advantages of cohort studies include the ability to assess multiple outcomes of a single exposure, evaluate the effects of rare exposures, and allow direct estimation of the incidence of an outcome in the exposed and unexposed populations. However, prospective cohort studies are relatively expensive, may take an extended period of time to carry out, and may also require a large number of individuals to evaluate risk for the occurrence of rare events. Alternatively, retrospective studies are limited by the completeness and validity of information available for collection and analysis.

\section{Common Types of Error in Observational Studies}

An observed association between an exposure and outcome should not be accepted as causal. Alternative explanations, including chance, bias, or confounding, may exist and obscure a true association, or produce a spurious association. Chance results from random variation, while bias is a systematic error that exists as a result of inappropriate study design. For example, common sources of bias include inappropriate selection of a control group that is not representative of the source population in a case-control study (selection bias) and dissimilar quality of information obtained from the different study groups due to 
differences in procedures to elicit information from individuals in the different study groups (interviewer bias) or differential recall of exposure or events among the groups (recall bias).

The third alternative explanation for a noncausal association is confounding, which occurs when another factor (confounder) is independently associated with both the exposure and event of interest and may cause a spurious association or obscure a true association. In appropriately designed studies, the effect of confounding may be evaluated and controlled in the design or analysis of the study findings. One special type of confounding is confounding by indication. This can occur when the indication for drug therapy is related to the outcome under study, for instance, when patients with more severe disease are more likely to receive the drug than those with less severe disease. Thus, the true association between the drug and outcome of interest may be obscured. ${ }^{57}$ For example, patients at high risk of myocardial infarction may be more likely to receive anti-hypertensive agents, such as beta blockers and calcium channel blockers. The occurrence of myocardial infarction may therefore be increased in users of these agents compared to nonusers. However, the association may not be causal in nature, and may reflect the increased severity or likelihood of the illness under study among users. $^{58}$

\section{Experimental Studies}

Interventional studies or clinical trials are prospective studies that involve two or more treatment groups in which the investigator controls the allocation of the treatment. Clinical trials are more commonly conducted to evaluate beneficial effects (efficacy) of preventing or treating disease than are observational studies. With random assignment of treatment (randomized clinical trials), the groups are more likely to be comparable regarding potential confounding factors, including biases in the 
selection of patients for a particular therapy based on perceived severity of the disease, thus making observed associations more convincing. However, randomized clinical trials are expensive, and may be more difficult to design and conduct than observational studies especially in the geriatric population. Special effort may be necessary to ensure patient compliance and continued follow-up. In addition, ethical considerations may preclude the evaluation of many potential adverse drug events.

\section{Measures of Association}

To evaluate the association between drug exposure and a specific adverse drug event, two important measures of association are frequently used: the relative risk and the attributable risk.

The relative risk is the ratio of the incidence of an adverse drug event in persons exposed to a drug to the incidence in those not exposed to the drug. It may be the ratio of two cumulative incidences (risk ratio) or the ratio of two incidence rates (rate ratio). A relative risk greater than 1.0 indicates that the exposed group has a greater risk for the occurrence of an event than the unexposed group. A relative risk less than 1.0 indicates that the exposed group has a lower risk than the unexposed group. A relative risk of 1.0 indicates that the risks of the event are identical in the exposed and unexposed groups, suggesting that the exposure is not associated with the event of interest.

In a case-control study, the incidence of an event in the exposed and unexposed groups cannot be directly estimated. An estimate of relative risk, the odds ratio, can be estimated from the data obtained in case-control studies. The odds ratio is the ratio of the odds of exposure among cases with the event of interest to the odds of exposure among the controls without the event.

The attributable risk is the absolute difference between the incidence in persons exposed to a factor and the incidence in persons unexposed to a factor. It may be the difference between 
two cumulative incidences (risk difference) or the difference between two incidence rates (rate difference). The attributable risk describes the risk of an event in exposed persons that can be attributable to the exposure. An attributable risk of zero indicates that the risks of an event are identical in the exposed and unexposed groups, suggesting that a drug exposure and an adverse event are unrelated.

\section{Data Sources for Observational Pharmacoepidemiologic Studies}

Ad hoc epidemiologic studies may be performed to evaluate the use and consequences of drugs in the population. In these studies, individuals must be identified from appropriate sources and recruited for study. However, other routinely collected data sources may be used to perform pharmacoepidemiologic studies.

Automated health care databases are increasingly being used for pharmacoepidemiologic and health services research. These databases contain patient-level information from such sources as Medicaid programs, health maintenance organizations, aggregate general practitioner systems, governmental health services, and other health plan databases. Depending on the database, information on patient demographics, pharmacy records, outpatient and inpatient diagnoses, outpatient and inpatient laboratory tests and procedures, as well as enrollment information may be available. Potential advantages of these databases include rapid, efficient access to information on drugs and medical services on large populations with diverse exposures, in turn potentially resulting in decreased costs for the conduct of the study. In addition, some sources of bias, such as recall and interviewer bias, may be minimized. However, the quality and completeness of the data from these sources should be evaluated. Coding errors and inadequate documentation by providers may be problematic. Other potential disadvantages include limited or no 
information on potential confounders or exposures such as alcohol or tobacco use, inpatient drug exposures, and over-the-counter drug use. In addition, less severe diseases may not be consistently documented.

A number of other resources, including registry data, vital statistics records, and drug utilization data collected by national and commercial sources, may also facilitate pharmacoepidemiologic studies. The usefulness of these and other sources of data depends on the objectives of the proposed study. ${ }^{59}$

\section{Future Research Directions}

Pharmacoepidemiology provides a powerful tool for examining the impact of drug therapy on the geriatric patient population. Techniques used to examine drug utilization patterns in the elderly and adverse effects of specific medications have been widely utilized with regard to a number of drug classes.

Approaches to fully evaluate the economic impact of drug therapy in the geriatric population have been less well established. Similarly, techniques to validly and reliably assess the effects of drug therapies on the quality-of-life of elderly patients have not been adequately developed. These highly important areas will continue to pose the greatest challenges, as well as opportunities, in geriatirc pharmacoepidemiology.

\section{References}

1. Laporte J.R., Carne X., Vidal X., Moreno V., Juan J., Upper gastrointestinal bleeding in relation to previous use of analgesics and non-steroidal anti-inflammatory drugs, Lancet 337 (1991), 85-89.

2. Faulkner G., Prichard P., Somerville K., Langman M.J.S., Aspirin and bleeding peptic ulcers in the elderly, British Medical Journal 297 (1988), 1311-1313. 
3. Langman M.J.S., Coggon D., Spiegelhalter D., Analgesic intake and the risk of acute upper gastrointestinal bleeding, American Journal of Medicine 76(6A) (1983), 79-82.

4. Langman M.J.S., Weil J., Wainwright P., Risks of bleeding peptic ulcer associated with individual non-steroidal anti-inflammatory drugs, Lancet 343 (1994), 1075-1078.

5. Garcia Rodriguez L.A., Walker A.M., Perez S., Nonsteroidal antiinflammatory drugs and gastrointestinal hospitalizations in Sasketchewan: a cohort study, Epidemiology 3 (1992), 337-342.

6. Garcia Rodriguez L.A., Jick H., Risk of upper gastrointestinal bleeding and perforation associated with individual non-steroidal anti-inflammatory drugs, Lancet 343 (1994), 769-772.

7. Griffin M.R., Ray W.A., Schaffner W., Nonsteroidal antiinflammatory drug use and death from peptic ulcer in elderly persons, Annals of Internal Medicine 109 (1988), 359-363.

8. Weil J., Colin-Jones D., Langman M., Prophylactic aspirin and risk of peptic ulcer bleeding, British Medical Journal 310 (1995), 827-830.

9. Solomon D.H., Gurwitz J.H., Toxicity of nonsteroidal antiinflammatory drugs in the elderly: is advanced age a risk factor?, American Journal of Medicine 102 (1997), 208-215.

10. Gurwitz J.H., Avorn J., Ross-Degnan D., Lipsitz L.A., Nonsteroidal anti-inflammatory drug-associated azotemia in the very old, Journal of the American Medical Association 264 (1990), 471-475.

11. Ray W.A., Griffin M.R., Downey W., Benzodiazepines of long and short elimination half-life and the risk of hip fracture, Journal of the American Medical Association 262 (1989), 3303-3307.

12. Gales B.J., Menard S.M., Relationship between the administration of selected medications and falls in hospitalized elderly patients, Annals of Pharmacotherapy 29 (1995), 354-358.

13. Herings R.M., Stricker B.H., de Boer A., Bakker A., Sturmans F., Benzodiazepines and the risk of falling leading to femur fractures. Dosage more important than elimination half-life, Archives of Internal Medicine 155 (1995), 1801-1807.

14. Avorn J., Gurwitz J.H., Bohn R.L., Mogun H., Monane M., Walker A., Increased incidence of levodopa therapy following metoclopramide use, Journal of the American Medical Association 274 (1995), 1780-1782. 
15. Gurwitz J.H., Avorn J., The ambiguous relation between aging and adverse drug reactions, Annals of Internal Medicine 114 (1991), 956-966.

16. Boston Collaborative Drug Surveillance Program, Regular aspirin intake and acute myocardial infarction, British Medical Journal 1 (1974), 440-443.

17. The Steering Committee of the Physician's Health Study Research Group, Final report on the aspirin component of the ongoing Physician Health Study, New England Journal of Medicine 321 (1989), 129-135.

18. Grodstein F., Stampfer M.J., Manson J.E., Postmenopausal estrogen and progestin use and the risk of cardiovascular disease, New England Journal of Medicine 335 (1996), 453-461.

19. Stampfer M.J., Colditz G.A., Willett W.C., Postmenopausal estrogen therapy and cardiovascular disease. Ten-year follow-up from the Nurses' Health Study, New England Journal of Medicine 325 (1991), 756-762.

20. Sullivan J.M., Vander Zwaag R., Hughes J.P., Estrogen replacement and coronary artery disease. Effect on survival in postmenopausal women, Archives of Internal Medicine 150 (1990), 2557-2562.

21. Donahue J.G., Weiss S.t., Livingston J.M., Goetsch M.A., Greineder D.K., Platt R., Inhaled steroids and the risk of hospitalization for asthma, Journal of the American Medical Association 277 (1997), 887-891.

22. Lee D., Bergman U., Studies of drug utilization. In Strom B.L. (ed.), Pharmacoepidemiology, John Wiley and Sons, Chichester, England (1994), pp. 379-393.

23. Beers M., Avorn J., Soumerai S.B., Everitt D.E., Sherman D.S., Salem S., Psychoactive medication use in intermediate-care facility residents, Journal of the American Medical Association 260 (1988), 3016-3020.

24. Schneider L.S., Pollock V.E., Lyness S.A., A meta-analysis of controlled trials of neuroleptic treatment in dementia, Journal of American Geriatrics Society 38 (1990), 553-563.

25. Ray W.A., Griffin M.R., Schaffner W., Baugh D.K., Melton L.J., Psychotropic drug use and the risk of hip fracture, New England Journal of Medicine 316 (1987), 363-369. 
26. Avorn J., Monane M., Everitt D.E., Beers M.H., Fields D., Clinical assessment of extrapyramidal signs in nursing home patients given antipyschotic medication, Archives of Internal Medicine 154 (1994), 1112-1117.

27. Avorn J., Bohn R.L., Mogun H., Gurwitz J.H., Monane M., Everitt D.E., Neuroleptic drug exposure and treatment of parkinsonism in the elderly: a case-control study, American Journal of Medicine (1995); (in press).

28. Gurwitz J.H., Goldberg R.J., Gore J.M., Coronary thrombolysis for the elderly, Journal of the American Medical Association 265 (1991), 1720-1723.

29. Fibrinolytic Therapy Trialists' (FTT) Collaborative Group, Indications for fibrinolytic therapy in suspected acute myocardial infarction: collaborative overview of early mortality and major morbidity results from all randomized trials of more than 1,000 patients, Lancet 343 (1994), 311-322.

30. Topol E.J., Califf R.M., Thrombolytic therapy for elderly patients, New England Journal of Medicine 327 (1992), 45-47.

31. Dudley N.H., Burns E., The influence of age on policies for admission and thrombolysis in coronary care units in the United Kingdom, Age and Aging 21 (1992), 95-98.

32. Jacox A., Carr D.B., Payne R., Management of cancer pain: adults (quick reference guide No. 9; AHCPR publication No. 94-0593). Rockville, Maryland: Agency for Health Care Policy and Research (1994).

33. Cleeland C.S., Gonin R., Hatfield A.K., Pain and its treatment in outpatients with metastatic cancer, New England Journal of Medicine 330 (1994), 592-596.

34. Kalish S.C., Gurwitz J.H., Krumholz H.M., Avorn J., A costeffectiveness model of thrombolytic therapy for acute myocardial infarction, Journal of General Internal Medicine 10 (1995), 321-330.

35. Classen D.C., Pestotnik S.L., Evans R.S., Lloyd J.F., Burke J.P., Adverse drug events in hospitalized patients. Excess length of stay, extra costs, and attributable mortality, Journal of the American Medical Association 277 (1997), 301-306.

36. Bates D.W., Spell N., Cullen D.J., The costs of adverse drug events in hospitalized patients, Journal of the American Medical Association 277 (1997), 307-311. 
37. Croog S.H., Levine S., Testa M.A., The effects of antihypertensive therapy on the quality-of-life, New England Journal of Medicine 314 (1986), 1657-1664.

38. Testa M.A., Anderson R.B., Nackley J.F., Hollenberg N.K., Quality-of-Life Hypertension Study Group. Quality-of-life and antihypertensive therapy in men-a comparison of captopril with enalapril, New England Journal of Medicine 328 (1993), 907-913.

39. Bombardier C., Ware J., Russell I.J., Larson M., Chalmers A., Reed J.L., Auranofin therapy and quality of life in patients with rheumatoid arthritis, American Journal of Medicine 81 (1986), 565-578.

40. Liang M.H., Cullen K.E., Larson M., Measuring function and health status in rheumatic clinical trials, Clinics in Rheumatic Disease 9 (1983), 531-539.

41. Gelber R.D., Lenderking W.R., Cotton D.J., Quality-of-life evaluation in a clinical trial of zidovudine therapy in patients with mildly symptomatic HIV infection, Annals of Internal Medicine 116 (1992), 961-966.

42. McMillem C., Fiegl P., Metch B., Hayden K.A., Meyskens F.L., Crowley J., Quality of life end points in cancer clinical trials: review and recommendations, Journal of National Cancer Institution 81 (1989), 485-495.

43. Preistman T.J., Baum M., Evaluation of quality of life in patients receiving treatment for advanced breast cancer, Lancet 1 (1976), 899-901.

44. Sugarbaker P.H., Barofsky I., Rosenberg S.A., Gianola F.J., Quality of life assessment of patients in extremity sarcoma clinical trials, Surgery 91 (1982), 17-22.

45. Greenfield S., Nelson E.C., Recent developments and future issues in the use of health status assessment measures in clinical settings, Medical Care 30(suppl 5) (1992), MS23-MS41.

46. Kajii T., Kida M., Takahahi K., The effect of thalidomide intake during 113 human pregnancies, Teratology 8 (1973), 163-166.

47. Mellin G.W., Katzenstein M., The saga of thalidomide, New England Journal of Medicine 267 (1962), 1238-1244.

48. Tauggig H.B., A study of the German outbreak of phocomelia, Journal of the American Medical Association 180 (1962), 1106-1114. 
49. Geiling E.M.K., Cannon P.R., Pathogenic effects of elixir of sulfanilamide (diethylene glycol) poisoning, Journal of the American Medical Association 111 (1938), 919-926.

50. Wallerstein R.O., Condit P.K., Kasper C.K., Statewide study of chloramphenicol therapy and fatal aplastic anemia, Journal of the American Medical Association 208 (1969), 2045-2050.

51. Inman W.H.W., Study of fatal bone marrow depression with special reference to phenylbutazone and oxyphenbutazone, British Medical Journal 1 (1977), 1500-1505.

52. Strom B.L., Carson J.L., Morse M.L., The effect of indication on the risk of hypersensitivity reactions associated with zomepirac sodium and other nonsteroidal anti-inflammatory drugs, Arthritis and Rheumatism 30 (1987), 1142-1148.

53. Strom B.L., West S.L., Sim E., Carson J.L., The epidemiology of acute flank pain syndrome from suprofen, Clinical Pharmacology and Therapeutics 46 (1989), 693-699.

54. Wright P., Untoward effects associated with practolol administration. Oculomucocutaneous syndrome, British Medical Journal 1 (1975), 595-598.

55. Gurwitz J.H., Col N.F., Avorn J., The exclusion of the elderly and women from clinical trials in acute myocardial infarction, Journal of the American Medical Association 268 (1992), 1417-1422.

56. Sachs R.M., Bortnichak E.A., An evaluation of spontaneous adverse drug reaction monitoring systems, American Journal of Medicine 81(suppl 5B) (1986), 49-55.

57. Strom B.L., Melmon D.L., The use of pharmacoepidemiology to study beneficial drug effects. In Strom B.L. (ed.), Pharmacoepidemiology, John Wiley and Sons, Chichester, England (1994), pp. 452-454.

58. Walker A.M., Confounding by indication, Epidemiology 7 (1996), 335-336.

59. Strom B.L., How should we perform pharmocoepidemiology studies? Choosing among the available alternatives. In Strom B.L. (ed.), Pharmacoepidemiology, John Wiley and Sons, Chichester, England (1994), pp. 337-350. 